\title{
Comparison of Gelation Time and Polyalcohol Effect on Hydrogels from Domestic and Wild Silk Fibroins
}

\author{
Huijing Zhao, ${ }^{1}$ Siyong Xiong, ${ }^{2}$ Mingzhong Li, ${ }^{1}$ Qiang Zhang, ${ }^{1}$ and Guiyang Liu ${ }^{1,3}$ \\ ${ }^{1}$ National Engineering Laboratory for Modern Silk, College of Textile and Clothing Engineering, Soochow University, \\ No. 199 Ren'ai Road, Industrial Park, Suzhou 215123, China \\ ${ }^{2}$ Industry Promoting Department, Suzhou Science and Technology Service Center, No. 979 Renmin Road, Suzhou 215002, China \\ ${ }^{3}$ Department of Textile, Nantong Textile Vocational Technology College, No. 105 East Qingnian Road, Nantong 226007, China \\ Correspondence should be addressed to Mingzhong Li, mzli@suda.edu.cn
}

Received 20 August 2011; Revised 20 January 2012; Accepted 8 February 2012

Academic Editor: Wen-Hua Sun

Copyright ( 2012 Huijing Zhao et al. This is an open access article distributed under the Creative Commons Attribution License, which permits unrestricted use, distribution, and reproduction in any medium, provided the original work is properly cited.

Silk fibroin (SF) hydrogels were obtained from both domestic (Bombyx mori) and wild (Antheraea pernyi) silkworms from aqueous silk fibroin solutions at room temperature. The gelation time of the Antheraea pernyi (A. pernyi) SF solution was significantly shorter than that of the Bombyx mori (B. mori) SF solution. The secondary structures of the two kinds of hydrogels were also compared. In order to further reduce the gelation time, various amounts of polyethylene glycol (PEG) were blended with the silk fibroins of A. pernyi and B. mori. The gelation time of both A. pernyi SF and B. mori SF decreased with the increased amount of PEG. After freeze-drying, the hydrogels were characterized through X-ray diffraction (XRD), Fourier transform infrared spectroscopy (FT-IR), and Raman spectroscopy. Results showed that the addition of polyalcohol did not change the main secondary structure of the hydrogels. However, the addition of polyalcohol did reduce the gelation time and triggered additional formation of $\beta$-sheets.

\section{Introduction}

Hydrogels can maintain a distinct three-dimensional porous structure with mechanical and structural properties similar to that of many natural tissues and extracellular matrices (ECMs) and can be delivered in a minimally invasive manner [1]. Therefore, hydrogels are of interest for many biomedical applications such as tissue engineering scaffolds, controlled drug release devices, and biosensors [2]. Hydrogels can be made of either synthetic materials or naturally derived materials [3]. Among naturally derived materials, it is agreed that silkworm silk fibroin (SF) is one of the most promising biomaterials because of its excellent biocompatibility, biosafety, controllable biodegradation rates, processability, and mechanical properties [4]. Silkworm silk fibroin is excreted by domestic (B. mori) and wild (A. pernyi) silkworms. Domestic SF hydrogels are of interest for use as bonefilling biomaterials, wound dressing, and so on and have proved to be promising for healing efficacy [5-10]. Compared with B. mori SF, A. pernyi SF, the most familiar wild SF, has more advantages in terms of chemical activity and highaffinity interactions with mammalian cells [11]. A. pernyi SF is rich in amino acids having polarity and ionogenic pendant groups, which are the potential reactive sites of chemical reactions. In terms of amino acids, $A$. pernyi SF has more Ala, Asp, and Arg and less Gly than does B. mori. Further, A. pernyi SF also has abundant alkaline amino acids (Arg and His) and the tripeptide sequence Arg-Gly-Asp (RGD), which is known to be a cell integrin receptor and to mediate special interactions between mammalian cells. It has been demonstrated in some research that $A$. pernyi SF performs better in terms of cell adhesion, growth, and phenotypic maintenance than does B. mori SF [12]. Also, A. pernyi has bioproperties similar to collagen, such as cell adhesion, growth, and proliferation. Therefore, it is supposed that biomedical materials made of $A$. pernyi SF have better bioproperties than do those made of $B$. mori SF. However, biomaterials made of $A$. perny $i$ SF have not been studied as widely as those made of $B$. mori SF, especially in hydrogels. Because of their improved properties and better performance, introducing wild SF 
hydrogels as a potential biomaterial in tissue engineering is very necessary. Therefore, hydrogels made of $A$. pernyi SF were prepared and studied in the present study.

Through reviewing research articles about B. mori SF hydrogels, we found that the gelation time of SF hydrogels is excessively long unless nonphysiological treatments (such as low $\mathrm{pH}$, high temperature, or additives) are considered [6, 13-17]. The question we want to answer is do the $A$. pernyi SF hydrogels have a similar problem? This question prompted us to search for effective ways to reduce the gelation time of $A$. pernyi SF solutions. In addition to the methods of $\gamma$-ray irradiation, ultrasonication, and shearing, blending auxiliary materials like polyalcohol with SF has been considered an effective method to reduce gelation time because it induces the transition of SF from random coil and $\alpha$-helix structures to $\beta$-sheet structures [18]. Among polyalcohols, polyethylene glycol (PEG) is a linear polymeric material that is polymerized by glycol monomers. The presence of abundant oxethyl groups in the PEG molecule that are capable of forming hydrogen bonds with water makes PEG a highly water-soluble material. PEG is also a nontoxic, nonimmunogenic, and amphipathic polymer, and it possesses a variety of properties pertinent to biomedical and biotechnical applications [18-20] . Thus, adding PEG to A. pernyi SF solutions was selected as a method for reducing gelation time in this study. B. mori SF hydrogels with and without PEG were also prepared as control samples. A. pernyi and $B$. mori SF hydrogels were characterized after freezedrying through X-ray diffraction (XRD), Fourier transform infrared spectroscopy (FT-IR), and Raman spectroscopy. The effect of PEG on gelation time and the final secondary structure of the hydrogels were also studied.

\section{Materials and Methods}

2.1. Preparation of SF Aqueous Solutions. The process of preparing SF aqueous solution is described elsewhere [11, 21]. To be specific, cocoons of $A$. pernyi were boiled for $30 \mathrm{~min}$ in a $0.25 \mathrm{wt} \% \mathrm{Na}_{2} \mathrm{CO}_{3}$ aqueous solution to remove sericin. Degummed A. pernyi SF fibers were rinsed thoroughly with pure water. After oven drying $\left(60^{\circ} \mathrm{C}\right)$, they were dissolved in $\mathrm{Ca}\left(\mathrm{NO}_{3}\right)_{2} \cdot 4 \mathrm{H}_{2} \mathrm{O}$ (the liquor ratio was $1: 10$ ) at $105^{\circ} \mathrm{C}$ for $5 \mathrm{~h}$. Then, the mixed solution was dialyzed against flowing distilled water for $96 \mathrm{~h}$ to remove salt. The concentration of A. pernyi $\mathrm{SF}$ aqueous solution was about $1.78 \mathrm{wt} \%$, and it was then concentrated to $3.0 \mathrm{wt} \%$ through air drying.

B. mori silks were degummed by being boiled for $30 \mathrm{~min}$ in a $0.06 \mathrm{wt} \% \mathrm{Na}_{2} \mathrm{CO}_{3}$ aqueous solution. After being rinsed and oven dried $\left(60^{\circ} \mathrm{C}\right)$, the degummed B. mori SF fibers were dissolved in the triadic solvent $\mathrm{CaCl}_{2} \cdot \mathrm{CH}_{3} \mathrm{CH}_{2} \mathrm{OH} \cdot \mathrm{H}_{2} \mathrm{O}$ (mole ratio $=1: 2: 8$ ) at $72^{\circ} \mathrm{C}$ for $1 \mathrm{~h}$ with stirring; the liquor ratio of the triadic solvent was $1: 10$. This solution was dialyzed against flowing distilled water for $96 \mathrm{~h}$. The final concentration of B. mori SF solution was also about $3.0 \mathrm{wt} \%$.

2.2. Preparation of SF Hydrogels. PEG 600 was added to the two $3.0 \mathrm{wt} \% \mathrm{SF}$ solutions in ratios of $0,50,100,150$, $300,500,700$, and $900 \mathrm{wt} \%$ (PEG/SF) and gently stirred at room temperature to disperse the PEG homogeneously. The mixed solutions were then left to stand at room temperature. The formation of gels was determined by inserting a glass capillary into the mixed solutions; when the liquid level in the glass capillary stopped rising, the gelation time of each sample was recorded. In order to measure their structure, the hydrogels were then frozen at $-40^{\circ} \mathrm{C}$ and lyophilized.

2.3. X-Ray Diffraction (XRD). The hydrogel samples were made into powders and measured by X-ray diffractometer (X'PERT PRO MPD) with $\mathrm{Cu} \mathrm{K} \alpha$ radiation $(\lambda=1.5406 \AA$ ). The scanning speed was $2 \% \mathrm{~mm}$. The diffraction intensity curves with $2 \theta$ from 5 to $45^{\circ}$ were obtained.

2.4. Fourier Transform-Infrared (FT-IR) Spectroscopy. The hydrogel samples were made into powders, using $\mathrm{KBr}$ tabletting to prepare specimens. An FT-IR spectrophotometer (Nicolet Avatar-IR 360) was used to analyze the samples. All spectra were recorded by absorption mode at $2 \mathrm{~cm}^{-1}$ interval and in the wavelength range of $1800 \sim 400 \mathrm{~cm}^{-1}$.

2.5. Raman Spectroscopy. Raman spectra were obtained by Raman spectrometer (LabRam-1B) at a wavelength of $632.8 \mathrm{~nm}$, the resolution of which was $2 \mathrm{~cm}^{-1}$. The scanning time for solid specimens was $200 \mathrm{~s}$.

\section{Results and Discussion}

3.1. Gelation Time. It can be seen in Figures 1 and 2 that the original gelation time of $A$. pernyi SF was 69.3 hours, which is much shorter than that of $B$. mori SF (gelation time was 288.6 h). Pure A. pernyi SF can also gelate quicker than that of $B$. mori SF with $500 \mathrm{wt} \%$ PEG (gelation time is $103.1 \mathrm{~h}$ ). The structure of the SF hydrogels was stabilized due to the formation of $\beta$-sheets, which served as physical cross-links $[22,23]$. A. pernyi SF is rich in amino acids having polarity and ionogenic pendant groups, which more easily forms reactions with other polar molecules and functional groups. The movements and interactions between the SF molecules themselves and the PEG in the A. pernyi SF aqueous solution would be more violent than those in the $B$. mori SF aqueous solution. The transition of $\beta$-sheets would be therefore more rapid and much easier.

The gelation time of $A$. pernyi SF decreased with the addition of PEG, and it decreased to $38.0 \mathrm{~h}$ when the amount of PEG was $500 \mathrm{wt} \%$. A small amount of PEG (only 50\%) can reduce the gelation time of $A$. pernyi SF, whereas the gelation time of $B$. mori SF required the addition of as much as $300 \mathrm{wt} \%$ PEG to significantly reduce the gelation time. PEG was highly water-soluble once it was blended with the SF aqueous solution, forming abundant hydrogen bonds and breaking the hydration layer around the SF molecules. The molecules of SF and PEG started to move and collide with each other, which accelerated the aggregation of SF molecules and triggered the breaking and rebuilding of hydrogen bonds. Through this process, random coil and $\alpha$ helix structure were induced to transform into $\beta$-sheets. 


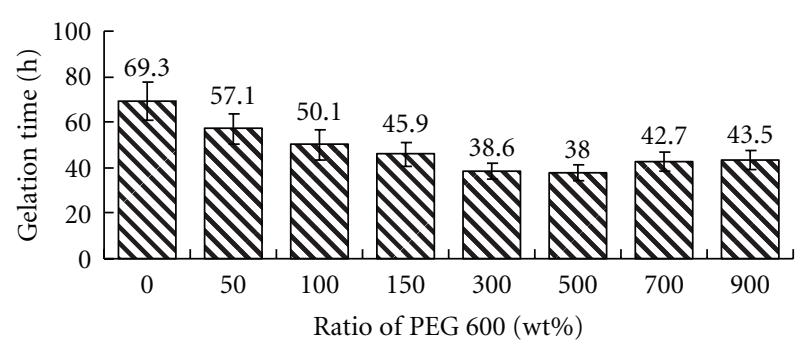

Figure 1: The relationship between gelation time of $A$. pernyi silk fibroin and PEG amount.

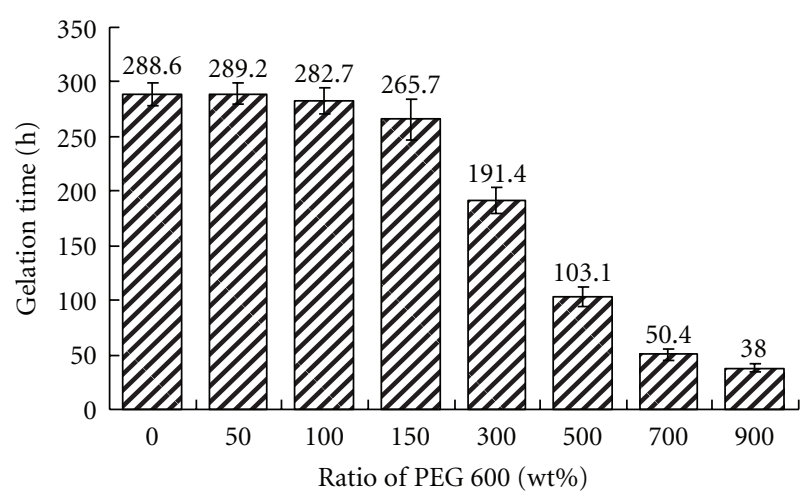

FIgURE 2: The relationship between gelation time of $B$. mori silk fibroin and PEG amount.

Figure 2 shows that preparing $B$. mori SF is timeconsuming, which is a drawback for the fabrication of SF hydrogels. However, in this study, it was found that A. pernyi SF can gelate much quicker than $B$. mori SF. Besides, adding a small amount of PEG (only 50\%) can further reduce the gelation time of $A$. pernyi SF. Hydrogels from A. pernyi SF also have better bioproperties and are more advantageous in terms of high-affinity interactions with cells; the shorter preparation time is another advantage for the use of these hydrogels in potential biomedical applications.

3.2. X-Ray Diffraction. The formation of $\beta$-sheets is an important issue in stabilizing the structure of SF hydrogels. Secondary conformation of hydrogels from pure $A$. pernyi SF, pure B. mori SF, and SFs blended with PEG are shown in the XRD curves in Figure 3. For the diffraction peaks of silk fibroin, please see $[21,24,25]$. In Figure $3(a)$, the diffraction peaks of pure A. pernyi SF hydrogels, shown in curve (A), appear around $16.5^{\circ}, 20.2^{\circ}, 24.9^{\circ}$, and $30.9^{\circ}$ and are attributed to $\beta$-sheet structure. The diffraction peaks at $16.5^{\circ}, 24.9^{\circ}$, and $30.9^{\circ}$ become wider (curve (B)) and then disappear (curve $(\mathrm{C})$ ), while the main diffraction peaks at $20.2^{\circ}$ remain. These results indicate that the secondary structure of $A$. pernyi SF hydrogels contains mainly $\beta$-sheets; however, with the addition of PEG (100 wt $\%$ and $300 \mathrm{wt} \%)$, the diffraction peaks of the hydrogels are covered up by those of PEG. A similar phenomenon is observed in Figure 3(b), in which the secondary structure of pure B. mori SF (curve (A), with diffraction peaks at $9.1^{\circ}, 20.7^{\circ}$, and $24.7^{\circ}$ ) consists mainly of $\beta$-sheets. With the addition of PEG, diffraction peaks at $9.1^{\circ}$ and $24.7^{\circ}$ are covered up, while peaks at $20.7^{\circ}$ remain (curves $(B)$ and $(C)$ ). The diffraction curves of the hydrogels with other blending ratios of SF and PEG were the same, so the data for these samples are not shown; only representative curves were selected for analysis. Figure 3 indicates that the secondary structure of both $A$. pernyi SF and $B$. mori SF are mainly $\beta$-sheets, and the addition of PEG did not influence the crystalline structure of the SF hydrogels.

3.3. FT-IR Spectroscopy. In order to prove the results of Xray diffraction, FT-IR spectra were also obtained in this study, the results of which are shown in Figure 4. For the characteristic peaks of silk fibroin in FT-IR spectra, see [25-28] . In Figure 4(a), relatively strong characteristic peaks appear in curve (A) at $1631 \mathrm{~cm}^{-1}$ (amide I), $1521 \mathrm{~cm}^{-1}$ (amide II), $1240 \mathrm{~cm}^{-1}$ (amide III), $965 \mathrm{~cm}^{-1}$ (amide IV), and $700 \mathrm{~cm}^{-1}$ (amide V), which means that pure $A$. pernyi SF hydrogels contain large amounts of $\beta$-sheets. Meanwhile, weak characteristic peaks appear in curve (A) at $881 \mathrm{~cm}^{-1}$ (amide IV) and $620 \mathrm{~cm}^{-1}$ (amide V), which indicates that a small amount of $\alpha$-helix structures coexist with the $\beta$ sheets. With the addition of PEG, the characteristic peaks attributed to $\beta$-sheets become stronger (curves (B) and (C)), while those attributed to $\alpha$-helix structures become smoother (curves (B) and (C)). A similar phenomenon is observed in Figure 4(b), in which the secondary structure of pure $B$. mori SF (curve (A)) consists of a large amount of $\beta$-sheets $\left(1635 \mathrm{~cm}^{-1}, 1620 \mathrm{~cm}^{-1}, 1234 \mathrm{~cm}^{-1}\right.$, and $\left.700 \mathrm{~cm}^{-1}\right)$ and a small amount of $\alpha$-helix structures $\left(610 \mathrm{~cm}^{-1}\right)$. With the addition of PEG, the characteristic peaks attributed to $\beta$ sheets become stronger (curves (B) and (C)), while those of the $\alpha$-helix $\left(610 \mathrm{~cm}^{-1}\right)$ disappear. The above phenomena indicate that the addition of polyalcohol triggered the transition of more $\beta$-sheets and that the main secondary structure in $A$. pernyi and B. mori SF hydrogels remains $\beta$-sheets.

3.4. Raman Spectroscopy. In order to confirm the results of X-ray diffraction and FT-IR, laser Raman spectroscopy of $A$. pernyi and B. mori SF hydrogels with added PEG at ratios of 0 and $300 \mathrm{wt} \%$ were detected, as shown in Figure 5. For the characteristic peaks of silk fibroin in Raman spectra, see [29]. In Figure 5(a), strong characteristic peaks in the Raman spectra of pure $A$. pernyi SF hydrogels (curve (A)) show around $1669.07 \mathrm{~cm}^{-1}, 1223.62 \mathrm{~cm}^{-1}$, and $1093.78 \mathrm{~cm}^{-1}$, which are attributed to $\beta$-sheets, while relatively weak characteristic peaks appear around $1657.88 \mathrm{~cm}^{-1}$, $1646.67 \mathrm{~cm}^{-1}, 1269.36 \mathrm{~cm}^{-1}$, and $907.29 \mathrm{~cm}^{-1}$, which are attributed to the $\alpha$-helix. Similarly, the secondary structure of pure $B$. mori SF hydrogels contains large amounts of $\beta$-sheets (strong characteristic peaks at $1665.99 \mathrm{~cm}^{-1}, 1235.64 \mathrm{~cm}^{-1}$, $1218.68 \mathrm{~cm}^{-1}$, and $1086.51 \mathrm{~cm}^{-1}$ ) and small amounts of $\alpha$ helix (relatively weak characteristic peaks at $1267.32 \mathrm{~cm}^{-1}$ and $1101.78 \mathrm{~cm}^{-1}$ ) and random coil (weak characteristic peaks at $1257.95 \mathrm{~cm}^{-1}$ ). With the addition of PEG, the characteristic peaks at $1646.67 \mathrm{~cm}^{-1}$ and $1269.36 \mathrm{~cm}^{-1}$ attributed to $\alpha$-helix disappear, whereas new characteristic peaks attributed to $\beta$-sheets $\left(1241.65 \mathrm{~cm}^{-1}\right.$ and $\left.1078.45 \mathrm{~cm}^{-1}\right)$ 


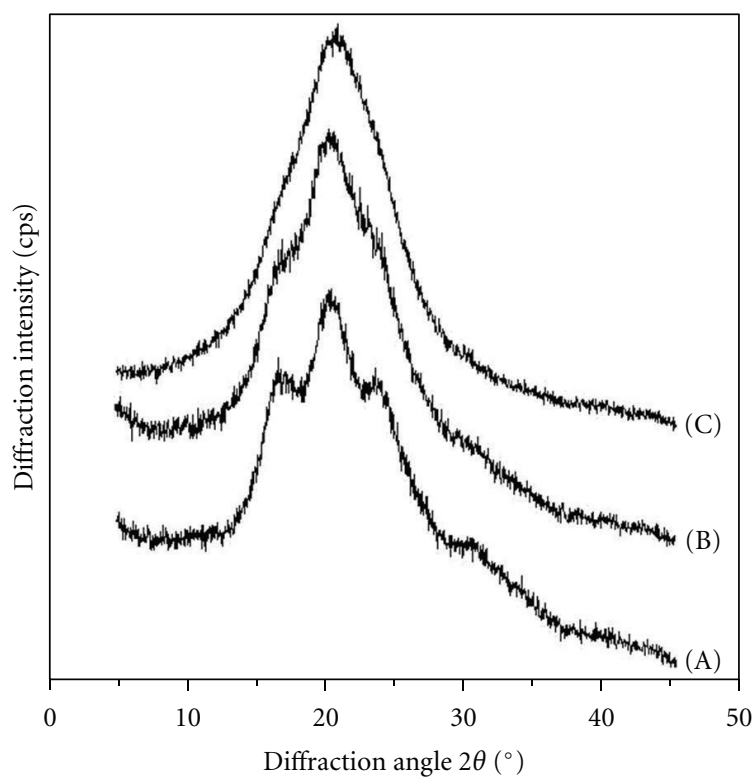

(a)

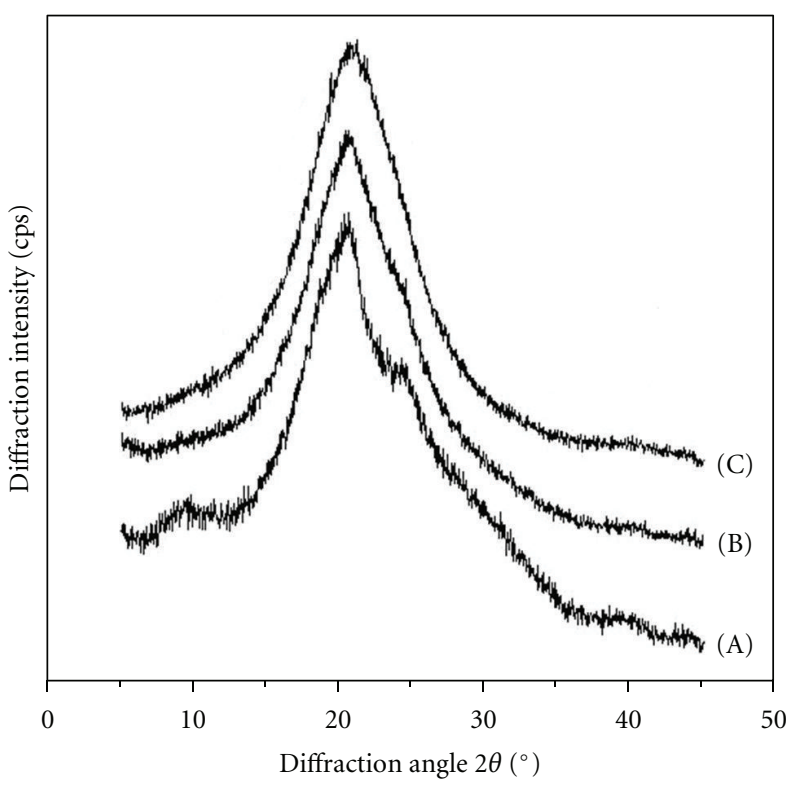

(b)

FIGURE 3: X-ray diffraction curves of A. pernyi silk fibroin hydrogels (a) and B. mori silk fibroin hydrogels (b), the ratio of PEG was 0 (A), $100 \mathrm{wt} \%(\mathrm{~B})$, and $300 \mathrm{wt} \%(\mathrm{C})$.

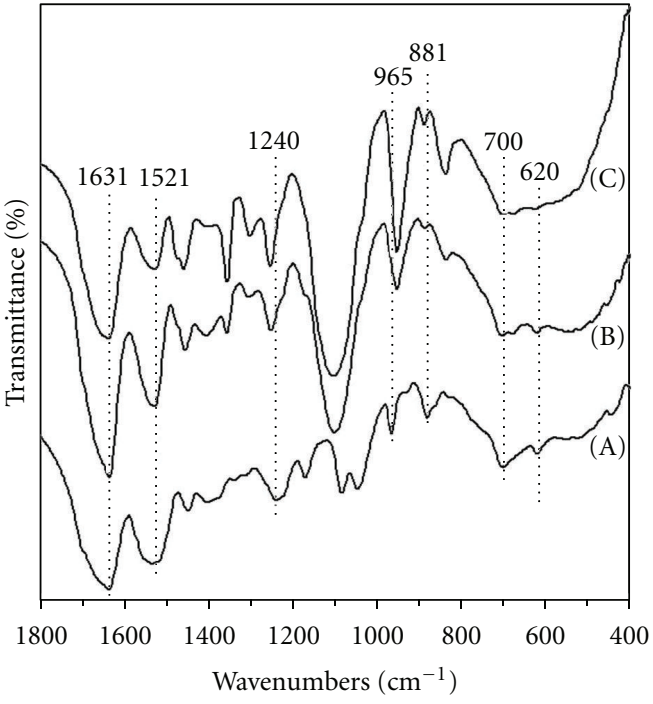

(a)

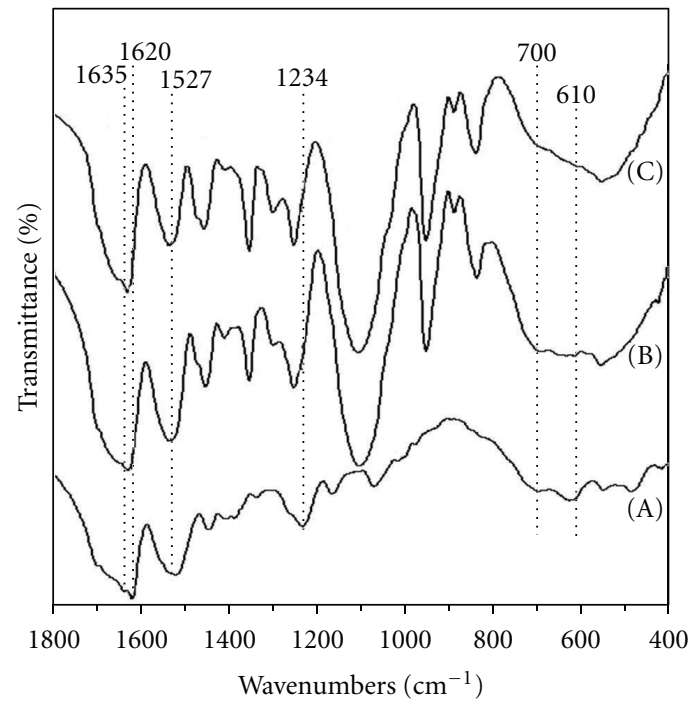

(b)

FIgure 4: FT-IR spectra of A. pernyi silk fibroin hydrogels (a) and B. mori silk fibroin hydrogels (b), the ratio of PEG was 0 (A), 100 wt\% (B), and $300 \mathrm{wt} \%$ (C).

appear in curve (B) of Figure 5(a). In Figure 5(b) curve (B), the characteristic peaks attributed to $\alpha$-helix $\left(1267.32 \mathrm{~cm}^{-1}\right.$ and $\left.1101.78 \mathrm{~cm}^{-1}\right)$ and random coil $\left(1257.95 \mathrm{~cm}^{-1}\right)$ disappear with the addition of PEG, while those of the $\beta$-sheets $\left(1666.99 \mathrm{~cm}^{-1}, 1240.30 \mathrm{~cm}^{-1}, 1086.51 \mathrm{~cm}^{-1}, 1064.22 \mathrm{~cm}^{-1}\right.$, and $1038.12 \mathrm{~cm}^{-1}$ ) show in the curve.

These results indicate that the main secondary structure in $A$. pernyi and B. mori SF hydrogels are $\beta$-sheets. With the addition of polyalcohol, more $\beta$-sheets in the secondary structure of both $A$. pernyi and B. mori SF hydrogels were formed. This is consistent with the results of X-ray diffraction and FT-IR.

Based on the above results and discussions, we would like to make some further points. Gelation occurs because SF chains tend to aggregate, passing from an amorphous (random coil) or unstable ( $\alpha$-helix) conformation to a more stable structure ( $\beta$-sheets). The reactive activity of $A$. pernyi $\mathrm{SF}$ is stronger than that of $B$. mori SF due to its molecular structure. Therefore, the transition to $\beta$-sheets in both the pure $A$. pernyi SF aqueous solution and the solutions blended 


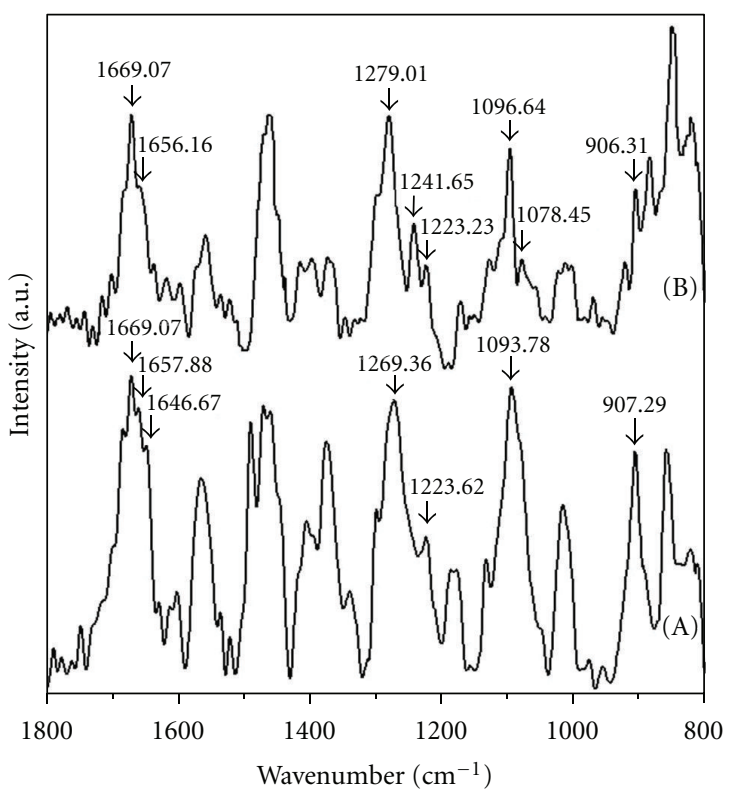

(a)

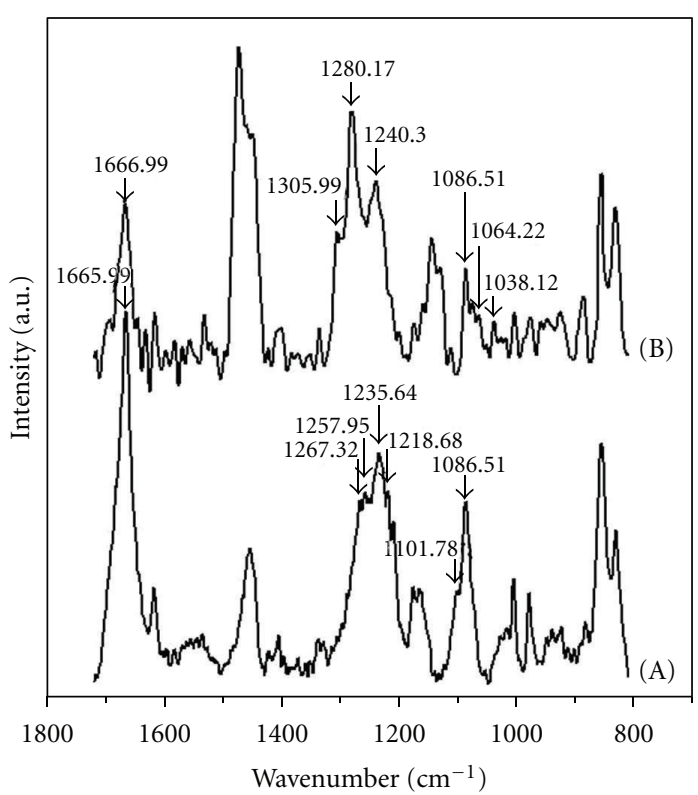

(b)

FIGURE 5: Raman spectra of A. pernyi silk fibroin hydrogels (a) and B. mori silk fibroin hydrogels (b), the ratio of PEG was 0 (a), 300 wt\% (b).

with PEG would be more rapid and much easier. Gelation of B. mori SF is both time- and energy-consuming, which has also been proven in other research $[6,7,24]$. In the present study, the gelation time of $A$. pernyi SF was proven to be much shorter than that of $B$. mori SF, even when nonauxiliary materials were added. Besides, adding even a small amount of PEG could reduce the gelation time. It has been proven that $A$. pernyi SF has better bioproperties than does $B$. mori SF. Therefore, A. pernyi SF hydrogels can be a more promising biomaterial.

The results of X-ray diffraction, FT-IR, and Raman spectroscopy indicate that the pure SF hydrogels (both A. pernyi and $B$. mori) contain mainly $\beta$-sheets, coexisting with a small amount of $\alpha$-helix and random coil structures. With the addition of PEG, the amount of $\alpha$-helix and random coil present is reduced, while more $\beta$-sheets are formed. This is probably because part of the $\alpha$-helix and random coil structures have been transformed into $\beta$-sheets. The addition of PEG also reduces the gelation time, as described previously. This would further indicate that, while the addition of polyalcohol did not influence the main secondary structure of the hydrogels, it did induce a quicker transition to $\beta$-sheets and triggered additional formation of $\beta$-sheets.

\section{Conclusions}

Hydrogels from pure A. pernyi SF, pure B. mori SF, and SF blended with PEG were obtained and their secondary structures were studied. The conclusions of this study are as follows. (1) The gelation time of pure A. pernyi SF was significantly shorter than that of $B$. mori SF. (2) The gelation time of $A$. pernyi SF can be reduced even more by adding a small amount of PEG. (3) The addition of polyalcohol did not influence the main secondary structure of the hydrogels.
However, it did induce a quicker transition to $\beta$-sheets and triggered additional formation of $\beta$-sheets.

In this study, hydrogels from wild (A. pernyi) SF were successfully prepared in a much shorter time compared to hydrogels prepared from domestic (B. mori) SF. Considering their better bioproperties, $A$. pernyi SF hydrogels are a promising biomaterial that can be applied in much wider biofields.

\section{Acknowledgments}

This work is supported by the National Natural Science Foundation of China (no. 30970714), Nature Science Foundation of Jiangsu Province (no. BK2010252), and Priority Academic Program Development of Jiangsu Higher Education Institutions.

\section{References}

[1] J. Kopeček, "Hydrogel biomaterials: a smart future?” Biomaterials, vol. 28, no. 34, pp. 5185-5192, 2007.

[2] J. L. Drury and D. J. Mooney, "Hydrogels for tissue engineering: scaffold design variables and applications," Biomaterials, vol. 24, no. 24, pp. 4337-4351, 2003.

[3] S. Van Vlierberghe, P. Dubruel, and E. Schacht, "Biopolymerbased hydrogels as scaffolds for tissue engineering applications: a review," Biomacromolecules, vol. 12, no. 5, pp. 13871408, 2011.

[4] C. Vepari and D. L. Kaplan, "Silk as a biomaterial," Progress in Polymer Science (Oxford), vol. 32, no. 8-9, pp. 991-1007, 2007.

[5] U. J. Kim, J. Park, C. Li, H. J. Jin, R. Valluzzi, and D. L. Kaplan, "Structure and properties of silk hydrogels," Biomacromolecules, vol. 5, no. 3, pp. 786-792, 2004. 
[6] X. Wang, J. A. Kluge, G. G. Leisk, and D. L. Kaplan, "Sonication-induced gelation of silk fibroin for cell encapsulation," Biomaterials, vol. 29, no. 8, pp. 1054-1064, 2008.

[7] M. Fini, A. Motta, P. Torricelli et al., "The healing of confined critical size cancellous defects in the presence of silk fibroin hydrogel," Biomaterials, vol. 26, no. 17, pp. 3527-3536, 2005.

[8] N. Guziewicz, A. Best, B. Perez-Ramirez, and D. L. Kaplan, "Lyophilized silk fibroin hydrogels for the sustained local delivery of therapeutic monoclonal antibodies," Biomaterials, vol. 32, no. 10, pp. 2642-2650, 2011.

[9] B. B. Mandal, S. Kapoor, and S. C. Kundu, "Silk fibroin/polyacrylamide semi-interpenetrating network hydrogels for controlled drug release," Biomaterials, vol. 30, no. 14, pp. 28262836, 2009.

[10] K. Numata, T. Katashima, and T. Sakai, "State of water, molecular structure, and cytotoxicity of silk hydrogels," Biomacromolecules, vol. 12, no. 6, pp. 2137-2144, 2011.

[11] W. Tao, M. Li, and C. Zhao, "Structure and properties of regenerated Antheraea pernyi silk fibroin in aqueous solution," International Journal of Biological Macromolecules, vol. 40, no. 5, pp. 472-478, 2007.

[12] Q. Fang, D. Chen, Z. Yang, and M. Li, "In vitro and in vivo research on using Antheraea pernyi silk fibroin as tissue engineering tendon scaffolds," Materials Science and Engineering C, vol. 29, no. 5, pp. 1527-1534, 2009.

[13] N. Bhardwaj, S. Chakraborty, and S. C. Kundu, "Freezegelled silk fibroin protein scaffolds for potential applications in soft tissue engineering," International Journal of Biological Macromolecules, vol. 49, no. 3, pp. 260-267, 2011.

[14] W. Xiao, J. He, J. W. Nichol et al., "Synthesis and characterization of photocrosslinkable gelatin and silk fibroin interpenetrating polymer network hydrogels," Acta Biomaterialia, vol. 7, no. 6, pp. 2384-2393, 2011.

[15] X. Hu, Q. Lu, L. Sun et al., "Biomaterials from ultrasonicationinduced silk fibroin-hyaluronic acid hydrogels," Biomacromolecules, vol. 11, no. 11, pp. 3178-3188, 2010.

[16] M. K. Yoo, H. Y. Kweon, K. G. Lee, H. C. Lee, and C. S. Cho, "Preparation of semi-interpenetrating polymer networks composed of silk fibroin and poloxamer macromer," International Journal of Biological Macromolecules, vol. 34, no. 4, pp. 263-270, 2004.

[17] G. M. Nogueira, M. A. De Moraes, A. C. D. Rodas, O. Z. Higa, and M. M. Beppu, "Hydrogels from silk fibroin metastable solution: formation and characterization from a biomaterial perspective," Materials Science and Engineering C, vol. 31, no. 5, pp. 997-1001, 2011.

[18] F. Cilurzo, C. G. M. Gennari, F. Selmin, L. A. Marotta, P. Minghetti, and L. Montanari, "An investigation into silk fibroin conformation in composite materials intended for drug delivery," International Journal of Pharmaceutics, vol. 414, no. 1-2, pp. 218-224, 2011.

[19] A. C. Sagle, H. Ju, B. D. Freeman, and M. M. Sharma, "PEGbased hydrogel membrane coatings," Polymer, vol. 50, no. 3, pp. 756-766, 2009.

[20] J. L. Roam, H. Xu, P. K. Nguyen, and D. L. Elbert, "The formation of protein concentration gradients mediated by density differences of poly(ethylene glycol) microspheres," Biomaterials, vol. 31, no. 33, pp. 8642-8650, 2010.

[21] M. Li, S. Lu, Z. Wu, K. Tan, N. Minoura, and S. Kuga, "Structure and properties of silk fibroin-poly(vinyl alcohol) gel," International Journal of Biological Macromolecules, vol. 30, no. 2, pp. 89-94, 2002.
[22] H. J. Jin and D. L. Kaplan, "Mechanism of silk processing in insects and spiders," Nature, vol. 424, no. 6952, pp. 1057-1061, 2003.

[23] A. Matsumoto, J. Chen, A. L. Collette et al., "Mechanisms of silk fibroin sol-gel transitions," Journal of Physical Chemistry B, vol. 110, no. 43, pp. 21630-21638, 2006.

[24] M. Li, W. Tao, S. Lu, and S. Kuga, "Compliant film of regenerated Antheraea pernyi silk fibroin by chemical crosslinking," International Journal of Biological Macromolecules, vol. 32, no. 3-5, pp. 159-163, 2003.

[25] M. Li, W. Tao, S. Kuga, and Y. Nishiyama, "Controlling molecular conformation of regenerated wild silk fibroin by aqueous ethanol treatment," Polymers for Advanced Technologies, vol. 14, no. 10, pp. 694-698, 2003.

[26] D. W. Jones, Introduction to the Spectroscopy of Biological Polymers, Academic Press, New York, NY, USA, 1976.

[27] W. K. Surewicz and H. H. Mantsch, "New insight into protein secondary structure from resolution-enhanced infrared spectra," Biochimica et Biophysica Acta, vol. 952, no. 2, pp. 115-130, 1988.

[28] X. Chen, Z. Shao, N. S. Marinkovic, L. M. Miller, P. Zhou, and M. R. Chance, "Conformation transition kinetics of regenerated Bombyx mori silk fibroin membrane monitored by timeresolved FTIR spectroscopy," Biophysical Chemistry, vol. 89, no. 1, pp. 25-34, 2001.

[29] P. Monti, G. Freddi, S. Sampaio, M. Tsukada, and P. Taddei, "Structure modifications induced in silk fibroin by enzymatic treatments. A Raman study," Journal of Molecular Structure, vol. 744-747, pp. 685-690, 2005. 

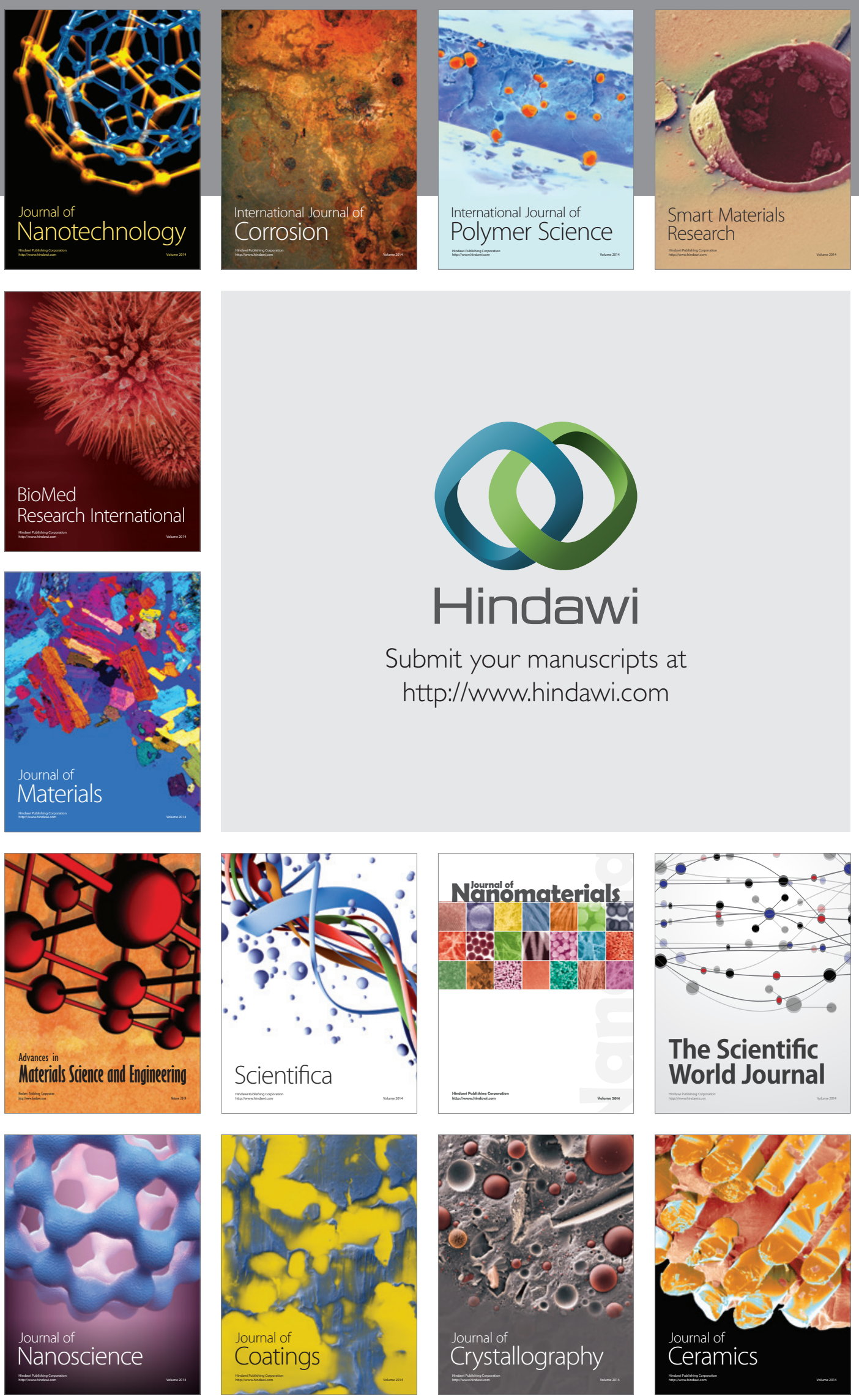

The Scientific World Journal

Submit your manuscripts at

http://www.hindawi.com

\section{World Journal}

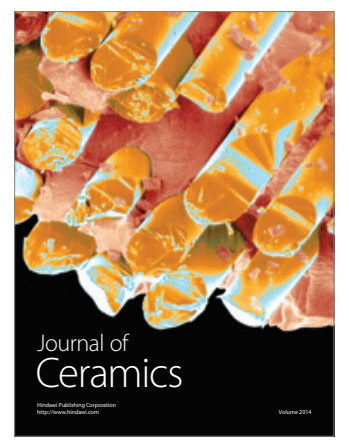

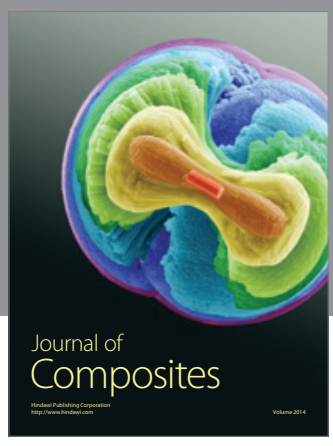
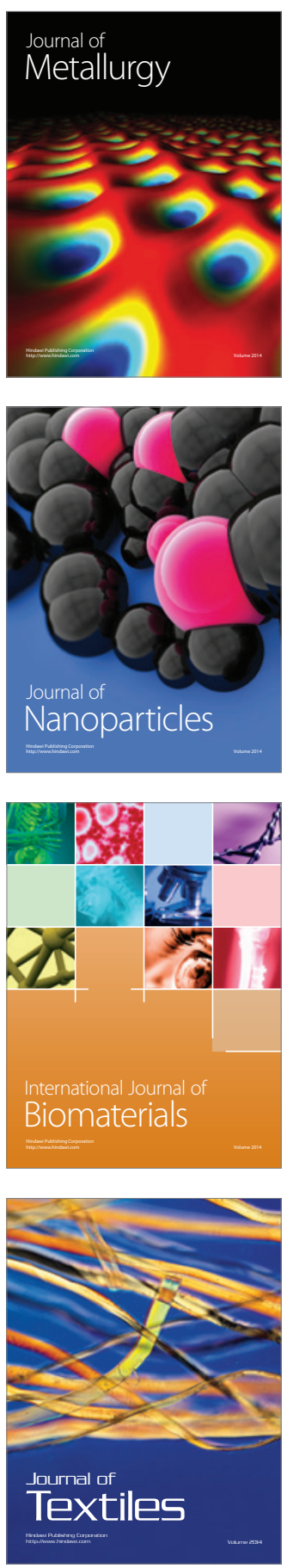